\title{
The impact of COVID-19 on U.S. adolescents: loss of basic needs and engagement in health risk behaviors
}

\author{
Akash Shroff $^{1}$ (D) . Julia Fassler ${ }^{1}$ (D) . Kathryn R. Fox ${ }^{2}$ D . Jessica L. Schleider ${ }^{1}$ D \\ Accepted: 17 October 2021 \\ (c) The Author(s), under exclusive licence to Springer Science+Business Media, LLC, part of Springer Nature 2021
}

\begin{abstract}
Across the United States, the COVID-19 pandemic created myriad challenges for youth and families, including losses of basic needs which may be associated with increased use of maladaptive coping behaviors. In a sample of 2491 U.S. youth (ages 13-16), demographic differences in loss of basic needs and maladaptive coping were assessed using regression models. More than $21 \%$ of adolescents endorsed losing one or more basic needs (e.g., loss of access to food or shelter) and $83 \%$ endorsed use of maladaptive coping strategies (e.g., using drugs or alcohol; self-harming behaviors) during the COVID-19 pandemic. Although adolescents with both majority and minority identities reported losing basic needs and engaging in maladaptive coping behaviors, minoritized youth reported more basic needs losses $(p s<0.05)$ and greater reliance on maladaptive coping strategies $(p s<.05)$ than their non-minoritized peers. Furthermore, adolescents who endorsed losing basic needs were more likely to also endorse engaging in maladaptive coping behaviors $(p<.05)$. Healthcare providers and teachers must consider basic need losses while structuring emotional and behavioral supports for youth during and beyond the COVID-19 pandemic.
\end{abstract}

Keywords Adolescents $\cdot$ COVID-19 $\cdot$ Mental health $\cdot$ Maladaptive coping $\cdot$ Loss of needs

\section{Introduction}

Since the start of the COVID-19 pandemic, families worldwide have undergone extreme financial hardship, sudden un- and under-employment, long-term social isolation, and intense distress. These compounding stressors have threatened to drastically increase risk for youth emotional and behavioral health challenges, including risk for maladaptive means of coping with pandemic-related distress-such as self-injury, substance use, and disordered eating behaviors. In China (Chen et al., 2020), Canada (Ellis et al., 2020), United Kingdom (Dewa et al., 2021), and Australia (Magson et al., 2021), youth have reported an increase in symptoms of depression, levels of anxiety, and stress related to the pandemic - regardless of variability in youth psychopathology pre-pandemic (Dewa et al., 2021). Furthermore, not all youth have been equally affected by the pandemic:

Jessica L. Schleider

jessica.schleider@stonybrook.edu

1 Department of Psychology, Stony Brook University, Stony Brook, NY, USA

2 Department of Psychology, University of Denver, Denver, CO, USA
Considering both individual (e.g. sex differences in psychopathology; Magson et al., 2021), and contextual risk factors (e.g., COVID-related barriers to accessing education; Kuhfeld et al., 2020) will be critical in identifying vulnerable subgroups of youth, both during and long after the pandemic. Therefore, the current study investigated demographic differences in loss of basic needs (e.g., food or housing insecurity) and maladaptive coping strategies (e.g., nonsuicidal self-injury, disordered eating, and over-exercise) as related to the pandemic, and the association between loss of basic needs and maladaptive coping, in a United States sample of adolescents.

\section{Loss of Basic Needs and Maladaptive Coping in Youth}

Maslow's Hierarchy of Needs states that individuals cannot achieve higher-level needs (i.e., esteem needs, self actualization) until basic needs (i.e., food and shelter) are met. Due to the pandemic, many adolescents were unable to consistently fulfill basic needs, undermining their capacity to fulfill higher-level needs as well. It is possible that these losses might have increased their reliance on maladaptive coping. For instance, poor family connectedness, one of Maslow's 
higher-level needs, predicts greater use of maladaptive coping (e.g., substance use and self-harm behaviors) in adolescents (Gervais \& Jose, 2020). Similar research suggests links between loss of basic needs, such as homelessness and food insecurity, and maladaptive coping strategies. For example, youth living in households with food insecurity-worrying about not being able to afford food or running out before obtaining more money-have shown greater likelihood of engaging in disordered eating behaviors than youth living in food-secure households (Oberle et al., 2019). Similarly, youth who experience homelessness (versus those with no homelessness history) more frequently reported engaging in substance use, binge drinking, and nonsuicidal self-injury (Cutuli, 2018). Furthermore, recent episodes of homelessness have predicted greater alcohol use and abuse in adolescents (Tompsett et al., 2013). The COVID-19 pandemic may increase the frequency of these kinds of environmental stressors, including food insecurity or homelessness, for a large number of families. As such, risk for associated adolescent behavioral and emotional difficulties may be important to monitor during and after the pandemic.

\section{Considering Minoritized Youth Identities}

Considerable literature indicates that structural, cultural, and identity-based stigma and discrimination place minoritized youth at heightened risk for behavioral health problems (Beccia et al., 2019; Blashill et al., 2021; Roulston et al., under review). For example, relative to youth with non-minoritized identities, sexual minority youth are more likely to report engaging in alcohol use (Phillips et al., 2017), disordered eating behaviors (Watson et al., 2017), and non-suicidal self-injury (Blashill et al., 2021). Having multiple marginalized identities, such as intersections between minoritized gender and race, has led to greater risk of such behaviors (Beccia et al., 2019). Compounding the deleterious effects on youth mental health on identity-based stigma and discrimination, minoritized youth are also at greater risk for loss of basic needs than their non-minoritized peers. For example, sexual minority youth are at higher risk for homelessness when compared to heterosexual youth (Corliss et al., 2011). Minoritized youth may face greater loss of basic needs in the context of the pandemic. Previous research has not examined rates of loss of basic needs and maladaptive coping separately for youth with diverse identities during the pandemic.

Recent work examining links between behavioral health problems and loss of basic needs has several limitations. Existing studies have been limited in their assessments of sociodemographic identities in youth, such as failing to measure gender identity and sexual orientation (Dewa et al., 2021), and have not included sufficiently large minoritized subgroups to examine racial/ethnic subgroup differences in risk behaviors. Often, race/ethnicity and other identityrelated factors have not been considered in research on disparities in youth mental health, especially in the context of the COVID-19 pandemic (Magson et al., 2021). Other limitations include reliance on parent reports of youth health-risk behaviors, despite the knowledge that parents are often unaware of maladaptive coping in their adolescent children, such as substance use, non-suicidal self injury and disordered eating (Bartholdy et al., 2017; Bell et al., 2021; Green et al., 2011; Klaus et al., 2009). For instance, research evaluating child eating behaviors and food insecurity has often included parent reported measures only, risking underreporting of the problem's prevalence (Oberle et al., 2019).

Much of clinical psychology and psychiatry research has focused on individual-level risk factors and characteristics that relate to emotional and behavioral problems. However, there is a clear need to also consider multilevel contextual factors-such as housing and food insecurity, physical safety, and access to education-in understanding risk for youth health risk behaviors. This contextual, multi-level focus is necessary to the long-term goal of promoting mental health equity for least-advantaged populations, per individual and contextual needs. For example, following disaster situations, using this model where youth risk for emotional and behavioral difficulties may be high. For instance, children exposed to at least one stressor related to Hurricane Katrina were found to have continued serious emotional disturbances, almost two years after the storm (Mclaughlin et al., 2009). Similar research indicates increases in youth substance use, aggression, and other behavioral health problems following natural disasters (McDermott \& Cobham, 2014). Parent psychopathology and sociodemographic factors are sometimes studied in the context of youth behavioral health problems following natural disasters. However, the relations of individual-level (e.g., race, gender, sexual orientation) and context-level factors (e.g., loss of basic needs) to youths' maladaptive coping behaviors has not been systematically explored since the start of the COVID-19 pandemic.

\section{The Current Study}

This study examined loss of basic needs (e.g., whether or not families had enough money for rent, transportation, or food) and use of maladaptive coping strategies (e.g., disordered eating, substance use, and self-injury) among a sample of diverse adolescents (ages 13-16) in the United States during the COVID-19 pandemic (November to December, 2020). Also, this study evaluated the prevalence of loss of basic needs, how youths' sexual, gender, and racial/ethnic identities relate to their loss of basic needs or their use of maladaptive coping behaviors, and whether youths who lost more basic needs reported using maladaptive coping strategies more frequently. 


\section{Method}

\section{Participants}

This pre-registered, secondary analysis study (https:// osf.io/ak5 $\mathrm{cp}$ ) used data from adolescents who completed the baseline (pre-intervention) survey battery within a web-based randomized controlled trial (NCT04634903; Schleider et al., 2021). Adolescents qualified for the study if they: (1) self-identified as being between 13 and 16 years old (inclusive) at the time of enrollment; (2) reported comfort reading and writing in English; (3) had access to the internet and a computer, laptop, or smartphone; and (4) endorsed elevated depressive symptoms, per an eligibility screener score of $>=2$ on the Patient Health Questionnaire-2 item version (PHQ-2; Richardson et al., 2010). All study procedures were approved by the University IRB prior to participant enrollment, and all adolescents provided online assent prior to beginning the study.

The final analytic sample included 2491 adolescent participants. Demographic information is reported in Table 1. Most (88.1\%) reported being assigned female sex at birth, and most identified as cisgender (70.7\%). A total of $961(38.6 \%)$ as bisexual or pansexual, 503 participants (20.2\%) identified as heterosexual, $254(10.2 \%)$ as gay/ lesbian/homosexual, $250(10.0 \%)$ as unsure or questioning, $235(9.4 \%)$ as no label or other, $152(6.1 \%)$ as queer, 126 $(5.1 \%)$ as asexual, and $10(0.4 \%)$ did not report. Regarding race and ethnicity, 1371 participants $(55.0 \%)$ identified as White/Cacasian, 360 (14.5\%) as Multi-Racial/MultiEthnic, 309 (12.4\%) as Hispanic/Latinx, $210(8.4 \%)$ as Asian, 165 (6.6\%) as Black/African American, 29 (1.2\%) as Other, $14(0.6 \%)$ as American Indian/Alaskian Native, 9 $(0.4 \%)$ as Native Hawaiian/Pacific Islander, and 24 (1.0\%) did not report. Adolescents in this study lived across all 50 states in the U.S. and Washington D.C., with the largest number of participants living in California (10.84\%), Texas $(8.07 \%)$, Florida $(6.06 \%)$, New York $(4.66 \%)$, Michigan (3.61\%), and Indiana (3.37\%). Finally, the mean Child Depression Inventory - Short Form (Kovacs, 2011) score in the sample was 14.23 and $81.2 \%$ of youth had a score $>=10$.

\section{Procedure}

Participants learned about the study through Instagram advertisements (total costs $=\$ 1279.11)$, which followed established ethics guidelines for passive, social mediabased recruitment (Gelinas et al., 2017). Posts included invitations to determine eligibility for a confidential, online psychology study, for which participants could earn
Table 1 Sample Demographics

Overall

$(\mathrm{N}=2491)$

\begin{tabular}{|c|c|}
\hline \multicolumn{2}{|l|}{ Age (years) } \\
\hline $13 / 14$ & $616(24.7 \%)$ \\
\hline $15 / 16$ & $1875(75.3 \%)$ \\
\hline \multicolumn{2}{|l|}{ Sex } \\
\hline Female & $2194(88.1 \%)$ \\
\hline Male & $256(10.3 \%)$ \\
\hline Missing & $41(1.6 \%)$ \\
\hline \multicolumn{2}{|l|}{ Gender } \\
\hline Cisgender & $1760(70.7 \%)$ \\
\hline Gender Minority & $731(29.3 \%)$ \\
\hline \multicolumn{2}{|l|}{ Race/Ethnicity } \\
\hline White/Caucasian & $1371(55.0 \%)$ \\
\hline American Indian/Alaskan Native & $14(0.6 \%)$ \\
\hline Asian & $210(8.4 \%)$ \\
\hline Black/African American & $165(6.6 \%)$ \\
\hline Hispanic/Latinx & $309(12.4 \%)$ \\
\hline Multi-Racial/Multi-Ethnic & $360(14.5 \%)$ \\
\hline Native Hawaiian/Pacific Islander & $9(0.4 \%)$ \\
\hline Other & $29(1.2 \%)$ \\
\hline Missing & $24(1.0 \%)$ \\
\hline \multicolumn{2}{|l|}{ Sexual Orientation } \\
\hline Heterosexual & $503(20.2 \%)$ \\
\hline Queer & $152(6.1 \%)$ \\
\hline Unsure/Questioning & $250(10.0 \%)$ \\
\hline No label/Other & $235(9.4 \%)$ \\
\hline Bisexual/Pansexual & $961(38.6 \%)$ \\
\hline Asexual & $126(5.1 \%)$ \\
\hline Gay/Lesbian/Homosexual & $254(10.2 \%)$ \\
\hline Missing & $10(0.4 \%)$ \\
\hline
\end{tabular}

Age, sex, race/ethncity, gender orientation, and sexual orientation categories were mutually exclusive

up to \$20 USD in gift cards across a 3-month period. Instagram posts linked to a Qualtrics survey, the first page of which included details about the project and an invitation to complete an eligibility screener. Eligible adolescents then reviewed an online assent form inviting them to participate. In line with ethical guidelines, given that it is difficult if not impossible to ensure parent/guardian consent online, parent permission was not required to participate in this study (waived by the University IRB; Smith et al., 2021). This provided anonymity and minimized access barriers (e.g., discomfort disclosing psychological distress, as parents are often unaware of their adolescents' depressive symptoms, including suicidal and morbid ideation, in up to $80 \%$ of cases; Brahmbhatt \& GruppPhelan, 2019). Adolescents could initiate the study at any time and location, using any internet-equipped device 
(smartphone, laptop, tablet). After starting the study, participants completed a battery of self-report questionnaires; the full measurement battery is listed in the original trial registration (NCT04634903); study-relevant items are detailed below. After, all participants were provided with mental health resources and an optional electronic safety plan. Recruitment and data collection took place between November and December of 2020. To note, the calculation of the loss of basic needs variable deviated from the preregistration. After inspecting the response text for "other" loss of basic needs, it was determined that participant responses were not identifying basic needs (i.e. "someone in my family had COVID"). Therefore, the "other" loss of basic needs were omitted in calculations.

\section{Measures}

\section{Demographic Information}

Participants were asked to report several demographic characteristics. Age was reported as an open ended numeric response and operationalized as two groups (13-14 and 15-16). Sex assigned at birth was reported as one category from male, female, other, or prefer not to say. Gender identity was reported as man/boy, woman/girl, female to male transgender/FTM, male to female transgender/MTF, trans male/trans masculine, trans female/trans feminine, genderqueer, gender expansive, androgynous, nonbinary, twospirited, third gender, agender, not sure or other. Youth were allowed to select multiple categories for gender identity; for the purposes of this study, participants were categorized as cisgender (i.e., gender matched birth assigned sex) or gender minority (i.e., any other gender). Race/ethnicity was reported as White/Caucasian, Black/African American, Asian, American Indian/Alaskian Native, Native Hawaiian/ Pacific Islander, Hispanic/Latinx, or Other. Youth could select multiple categories for race/ethnicity and were categorized as multi-racial/multi-ethnic if they chose more than one category. Sexual orientation was reported as one category for heterosexual/straight, gay/lesbian/homosexual, bisexual, pansexual, asexual, unsure/questioning, no label, other/not listed, or queer. From their responses, participants' were then categorized as hetersexual, homosexual, bisexual/ pansexual, asexual, queer, unsure/questioning, or no label/ other.

\section{Loss of Basic Needs}

Participants were asked to self-report on how the COVID19 pandemic affected them or their family in the past two weeks. Item choices were adapted from those included in the CDC's publicly available item bank for research on COVID-19 (CDC, 2020). Items were rated 1 ("Yes") or 0
("No") for the following statements, "Family did not have enough money to pay rent," "Family did not enough money for gas/transportation," "Family did not have enough money for food," "Family did not have a regular place to sleep or stay," "I could not attend school in person," "I could not attend school at all," and "other". The calculation for the loss of basic needs did not include "I could not attend school in person" or "other" as a loss of basic needs because majority of schools offered virtual instruction and, after reviewing the open-ended responses for "other", it was determined that these responses were not related to basic needs (e.g., "my family member has COVID" or "I can't see my friend"). Loss of basic needs was operationalized as a count variable, ranging from 0 basic needs lost to 5 basic needs lost.

\section{Maladaptive Coping Behaviors}

To assess coping behaviors, participants were asked to indicate which behaviors they had engaged in to cope with social distancing and isolation due to the COVID-19 pandemic, since the pandemic began. Item choices were adapted from those included in the CDC's publicly available item bank for research on COVID-19 (CDC, 2020). For the present study, only items related to maladaptive coding (rated 1 ("Yes") or 0 ("No")) for the following coping behaviors: "Smoking more cigarettes or vaping more," "Drinking alcohol," "Using cannabis or marijuana," "cutting or self-injury," "Over-exercise," "Eating more food than usual," and "Eating less food than usual." Other coping behaviors, not included in this composite, involved options such as "making time to relax" or "contacting a healthcare provider" were included. Maladaptive coping was operationalized as a count variable ranging from 0 maladaptive behaviors present to 7 such behaviors present.

\section{Data Analysis}

Analyses and data visualizations were performed in $\mathrm{R}(\mathrm{R}$ Core Team, 2021) via the tidyverse (Wickham et al., 2019), purrr (Henry \& Wickham, 2020), stats (R Core Team, 2021), MASS (Venables \& Ripley, 2002), psych (Revelle, 2020), ggplot (Wickham et al., 2020), corrplot (Wei \& Simko, 2021), and Table 1 (Rich, 2021) packages. The mean and the variance for loss of basic needs and maladaptive coping behaviors were first calculated. The variance was greater than the mean count of loss of basic needs, indicating that the data were overdispersed; moreover, most did not indicate a loss of basic needs, indicating the data were zero-inflated. Based on these criteria, the data were fit to a negative binomial regression model to gauge whether total number of basic needs lost varied by sociodemographic variables (e.g., 13-14 versus 15-16 years; female versus male sex; cisgender versus gender minority identity; heterosexual 
versus sexual minority identities; identifying as White versus Black, Asian, Multiracial, Latinx, American Indian/ Alaskan Native, or Native Hawaiian/Pacific Islander). To calculate engagement in maladaptive coping behaviors across demographics a poisson regression model was used, because the mean and variance in the count of maladaptive coping behaviors were equal, and because the data were not zero-inflated. For all analyses, alpha was set to $p<0.05$ and Holm-Bonferroni corrections were applied to adjust for multiple comparisons. A chi-square test was used to determine the association between youths' self-reported total loss of basic needs and the number of maladaptive coping behaviors they engaged in since the start of the pandemic. A spearman's rank order correlation was used to assess the link between total number of basic needs lost and total number of maladaptive coping behaviors endorsed.

Exploratory, post-hoc sensitivity analyses were conducted to determine whether patterns observed regarding links between loss of basic needs and all maladaptive coping behaviors, considered together, persisted when excluding eating-related maladaptive behaviors (i.e., eating more/ less to cope with the pandemic), which may in some cases occur as a direct result of basic needs loss (e.g., not having enough money for food or housing). Specifically, the analyses described above were replicated but used an alternative "total maladaptive coping behaviors" variable, reflecting a count of all maladaptive behaviors except for "eating more/ less than usual." The alternative "total maladaptive coping behaviors" variable scores ranged from 0 to 5 . All of the code for the analyses is available at https://osf.io/3qtcb/.

\section{Results}

\section{How Common Were Loss of Basic Needs and Use of Maladaptive Coping Behaviors during the COVID-19 Pandemic among U.S. Adolescents?}

Of the 2491 youth in the study sample, $526(21.1 \%)$ reported losing at least one basic need during the pandemic (Fig. 1). Specifically, 10.9\% $(\mathrm{N}=261)$ reported that their family did not have enough money for food, $8.9 \%$ $(\mathrm{N}=214)$ reported that their family did not have enough money for rent/housing, and $6.8 \%(\mathrm{~N}=164)$ reported loss of access to needed transportation. Further, $1.4 \%$ of youth $(\mathrm{N}=33)$ reported that their family did not have a regular place to live, and $8.1 \%(\mathrm{~N}=195)$ reported that they could not attend school at all for an extended period of timewhether school was in-person or virtual.

Maladaptive coping behaviors were extremely common in the sample, with 2077 of $2491(83.4 \%)$ endorsing at least one such behavior (Fig. 2). Behaviors endorsed by youth included those related to eating patterns $(51.4 \%$, $\mathrm{N}=1233$ eating less than usual; $49.3 \%, \mathrm{~N}=1184$ eating more than usual), non-suicidal self-injury $(23.7 \%$, $\mathrm{N}=568)$, over-exercise $(9.6 \%, \mathrm{~N}=230)$, and non-prescription substance use (12.5\% marijuana, $\mathrm{N}=299 ; 11.1 \%$ smoking or vaping, $\mathrm{N}=267 ; 9.2 \%$ alcohol, $\mathrm{N}=222$ ).
Fig. 1 Loss of Basic Needs Related to the COVID-19 Pandemic

Youth $(\mathrm{N}=526$ out of 2491) in the United States endorsed loss of basic needs as related to the COVID-19 pandemic. Youth could endorse losing multiple basic needs simultaneously..

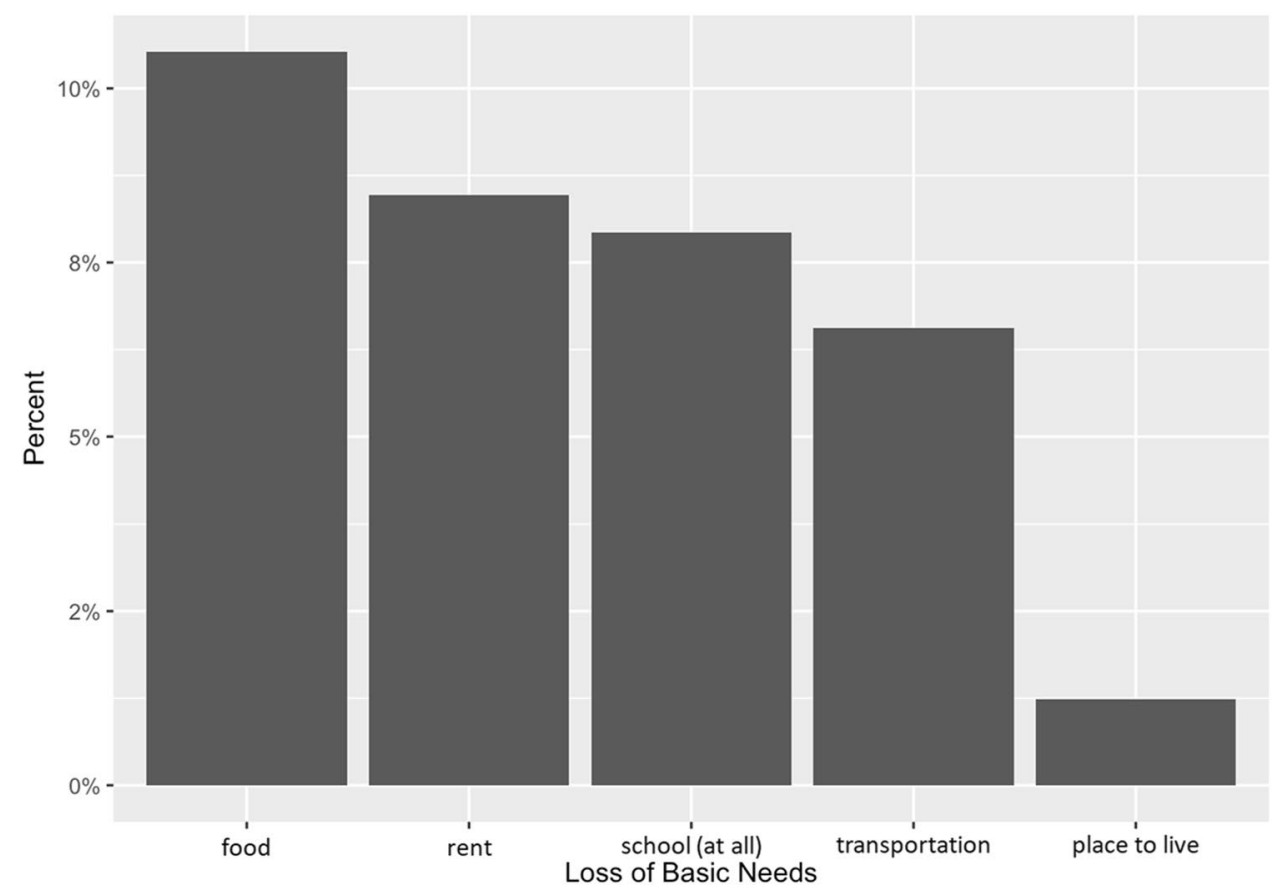




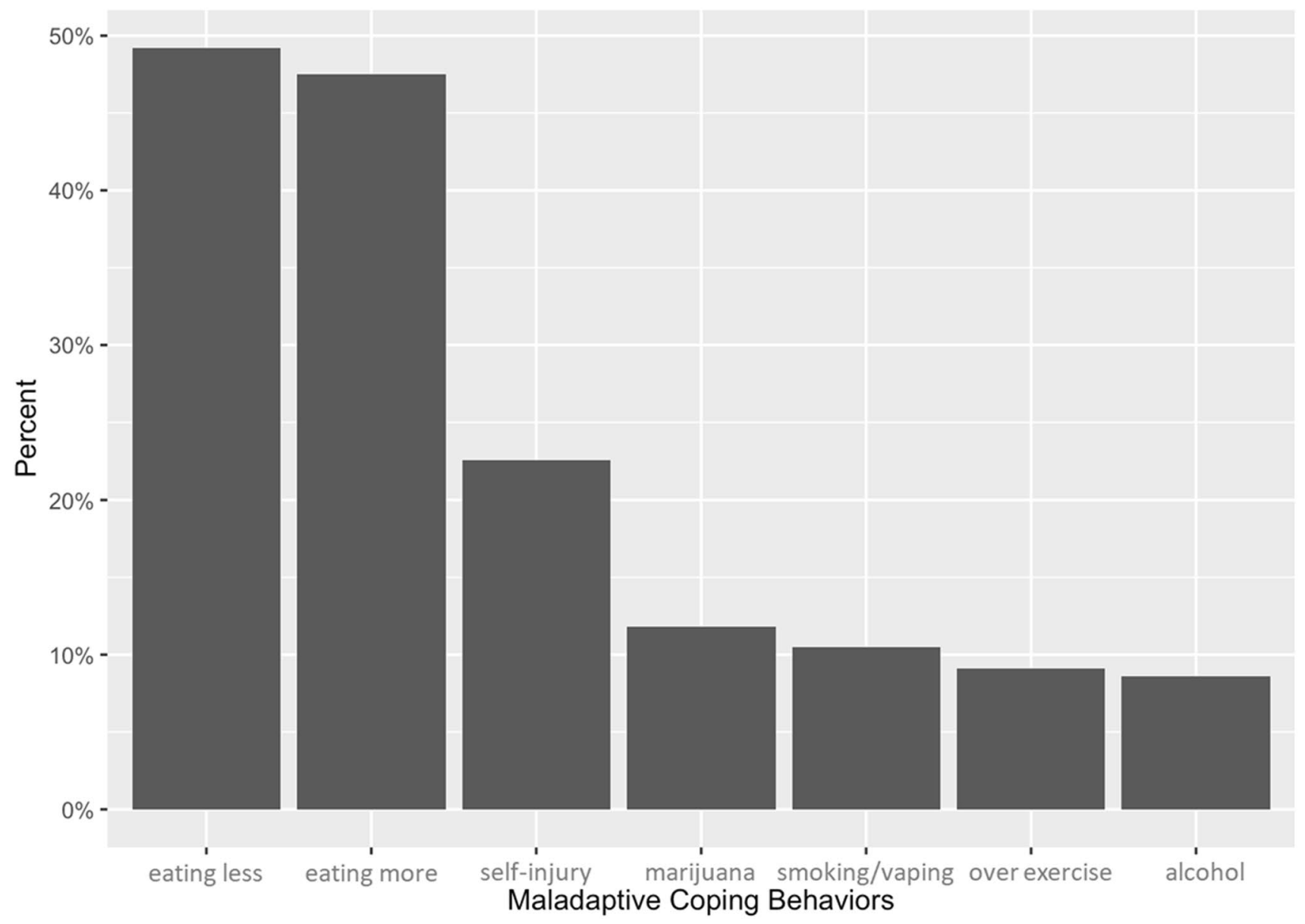

Fig. 2 Maladaptive Coping Behaviors Related to the COVID-19 Pandemic. Youth $(\mathrm{N}=2077$ of 2491) in the United States endorsed maladaptive coping behaviors as related to the COVID-19 pandemic.

\section{Did Youths' Sociodemographic Identities Relate to their Loss of Basic Needs or their Use of Maladaptive Coping Behaviors during the COVID-19 Pandemic?}

Per results of a negative binomial regression analysis, gender minority (versus cisgender) identity was associated with a $49.4 \%$ greater count of loss of basic needs, $p_{\text {holm }}=.0014, \mathrm{OR}=1.49$. A significant omnibus effect emerged for youth race/ethnicity $(p<.001)$. Post-hoc comparisons revealed that identifying as Multi-racial/Multiethnic identity was associated with a $71.4 \%$ greater count of loss of basic needs, $p_{\text {holm }}<.001, \mathrm{OR}=1.71$, compared to identifying as White/Caucasian; Hispanic/Latinx youth were $54.3 \%$ more likely than White/non-Hispanic youth to report basic needs loss, $p_{\text {holm }}=.0366, \mathrm{OR}=1.54$, compared to youth identifying as White/Caucasian. There were no significant differences in counts of loss of basic needs across age (13 to 16), biological sex (female versus male), or sexual orientation (heterosexual, homosexual, bisexual/
Self-injury was defined as non-suicidal self injury (i.e., self-injury without intent to die). Youth could endorse multiple maladaptive coping behaviors simultaneously.

pansexual, asexual, queer, unsure/questioning, versus no label/other).

Per results of a poisson regression analysis, gender minority (versus cisgender) identity was associated with a $22.8 \%$ greater count of maladaptive coping behaviors, $p_{\text {holm }}<.001, \mathrm{OR}=1.23$. A significant omnibus effect emerged for youth race/ethnicity $(p<.001)$. Post-hoc comparisons revealed that identifying as Asian indicated engaging in $37.1 \%$ fewer maladaptive coping behaviors, $p_{\text {holm }}<.001, \mathrm{OR}=0.63$, and African American indicated engaging in $20.6 \%$ fewer maladaptive coping behaviors, $p_{\text {holm }}=.0167, \mathrm{OR}=0.79$, compared to participants identifying as White/Cacuasian. Participants assigned male sex at birth indicated engaging in $26.9 \%$ less maladaptive coping behaviors, $p_{\text {holm }}<.001, \mathrm{OR}=0.73$, when compared to those assigned female. Sexual minority youth reported engaging in $41.2 \%$ more maladaptive coping behaviors compared to participants identifying as Heterosexual, $p_{\text {holm }}<.001, \mathrm{OR}=1.41$. Age was unrelated to counts of maladaptive coping behaviors. 
After conducting sensitivity analyses excluding eatingrelated maladaptive coping behaviors (which may in fact reflect loss of basic needs linked to food insecurity), sex differences in maladaptive coping became non-significant, whereas other disparities widened. Specifically, gender minority identity (versus cisgender identity) was associated with a $49.3 \%$ greater count of maladaptive coping behaviors, $p_{\text {holm }}<.001$, OR $=1.49$, after excluding eating-related coping behaviors from analyses. African American (versus Whitle/Caucasian) was associated with a $40.7 \%$ fewer count of maladaptive coping behaviors $p_{\text {holm }}=.0065$, OR $=0.59$. Similarly, Asian (versus White/Caucasian) was associated with a $67.8 \%$ fewer count of maladaptive coping behaviors $p_{\text {holm }}<.001$, $\mathrm{OR}=0.32$. A significant omnibus effect emerged for youth sexual orientation $(p<.001)$. Post-hoc comparisons revealed that identifying, in comparison to heterosexual participants, as queer $\left(166.9 \% ; p_{\text {holm }}<.001, \mathrm{OR}=2.67\right)$, bisexual/pansexual $\left(118.9 \%\right.$; $p_{\text {holm }}<.001$, OR $\left.=2.19\right)$, and homosexual $\left(75.1 \%\right.$; $\left.p_{\text {holm }}<.001, \mathrm{OR}=1.75\right)$ participants reported greater use of maladaptive coping behaviors. Similarly, participants who identified not using a label or other $\left(85.7 \%\right.$; $\left.p_{\text {holm }}<.001, \mathrm{OR}=1.86\right)$ and participants who identified as being unsure or questioning $\left(53.4 \% ; p_{\text {holm }}=.0199, \mathrm{OR}=1.53\right)$ reported greater use of maladaptive coping behaviors compared to heterosexual participants.

\section{Did Youths Who Lost more Basic Needs Report Using more Maladaptive Coping Strategies during the COVID-19 Pandemic?}

A chi-square test indicated a significant association between loss of more basic needs and engaging in a greater number of maladaptive coping behaviors during the COVID19 pandemic, $\chi^{2}(2, \mathrm{~N}=2491)=27.46, p_{\text {holm }}<.001$. For participants who reported losing one or more basic needs, $54.0 \%$ of participants indicated engaging in two or more maladaptive coping behaviors, $34.8 \%$ reported engaging in one, and $11.2 \%$ reported engaging in none. For participants that indicated no loss of basic needs, $42.1 \%$ reported two or more maladaptive coping behaviors, $39.8 \%$ reported engaging in one and $18.1 \%$ reported engaging in none.

Results were similar after removing eating behaviors from the measure of maladaptive coping. A chi-square test indicated a significant association between loss of more basic needs and engaging in a greater number of maladaptive coping behaviors during the COVID-19 pandemic, $\chi^{2}(2$, $\mathrm{N}=2491)=41.96, p_{\text {holm }}<.001$ (Fig. 3). For participants who reported one or more loss of basic needs, $52.7 \%$ engaged in no maladaptive coping behaviors, $23.2 \%$ reported engaging in one, $24.1 \%$ reported in two or more maladaptive coping behaviors. Also, for participants who reported no loss of needs, $63.4 \%$ reported no maladaptive coping behaviors, $23.7 \%$ reported engaging in one and $12.9 \%$ reported engaging in two or more. Moreover, Spearman's rank-order correlation confirmed a weak, positive relationship between loss of basic needs and maladaptive coping behaviors during the
Fig. 3 Maladaptive Coping Behaviors Across Loss and No Loss of Basic Needs. Footnote: Youth that endorsed at least one or more loss of basic needs were more likely to endorse engaging in at least one or more maladaptive coping behavior. Loss of basic needs and maladaptive coping behaviors were not mutually exclusive. (Top $=$ No loss; Bottom $=1+$ loss)

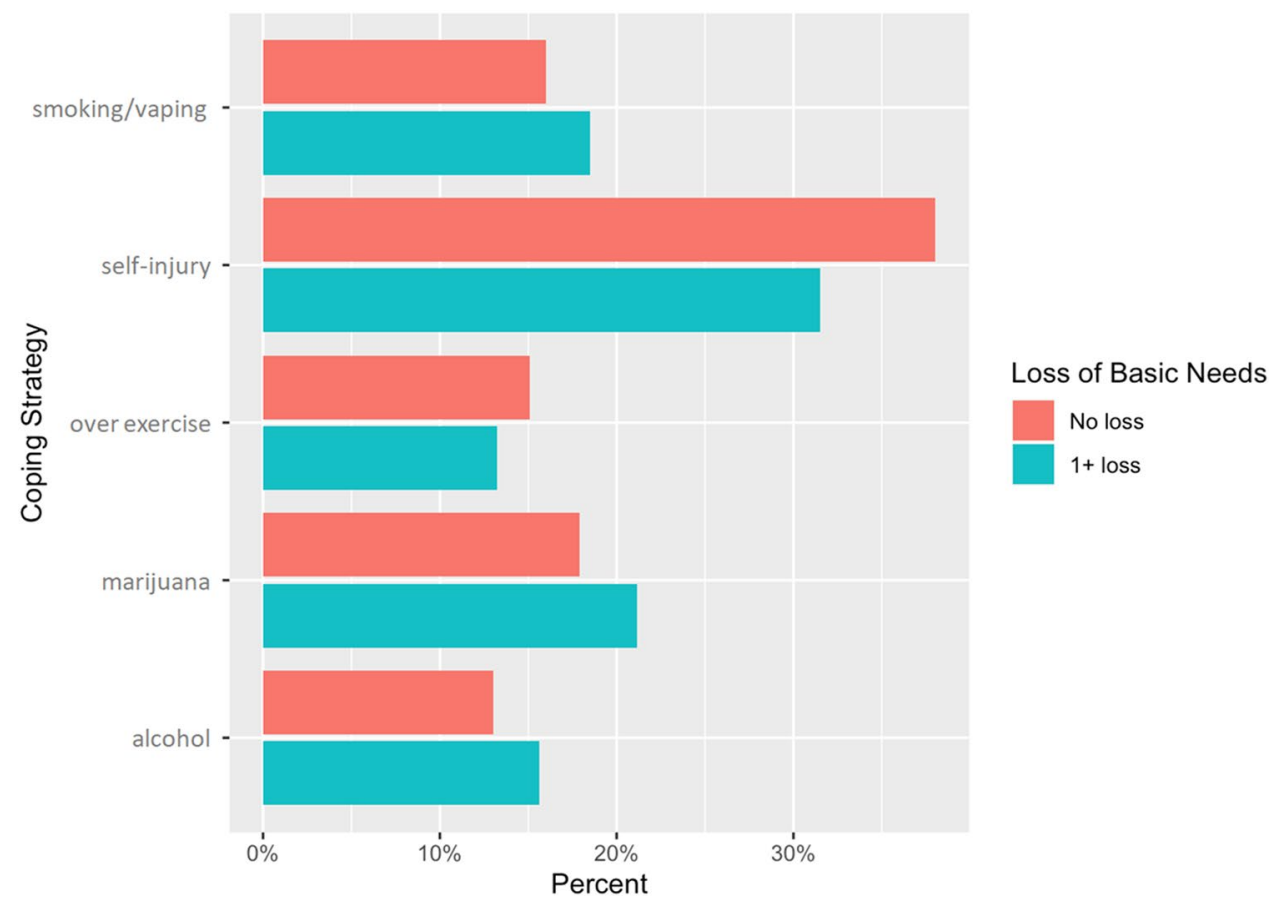


COVID-19 pandemic, $\mathrm{r}_{\mathrm{s}}=0.119, \mathrm{n}=2491, p_{\text {holm }}<.001$, after removing eating behaviors.

\section{Discussion}

Based on self-report data from 2491 adolescents across the United States, $21 \%$ of adolescents endorsed losing one or more basic needs and $83 \%$ endorsed use of maladaptive coping strategies during the COVID-19 pandemic. Although adolescents with diverse identities reported losing basic needs (e.g., loss of access to food or shelter) and engaging in maladaptive coping behaviors (e.g., using drugs or alcohol; self-harming behaviors), minoritized youth reported more basic needs losses and greater reliance on maladaptive coping strategies than their non-minoritized peers Furthermore, adolescents who endorsed losing basic needs were more likely to also endorse engaging in maladaptive coping behaviors.

Like many previous natural disasters, the COVID-19 pandemic led to a precipitous, wide-scale uptick in loss of basic needs among U.S. families (Chen et al., 2020; Dewa et al., 2021). For example, in the present study, half of adolescents who endorsed losing one or more basic needs also endorsed "not having enough money to buy food" at some point since the start of the pandemic - an alarming proportion, regardless of surrounding circumstances. In addition to loss of basic needs, many youth likely faced some combination of social isolation, familial financial strain, and worsened mental health as a result of the pandemic (Dewa et al., 2021). It is possible that youth may rely upon behaviors such as non-suicidal self-injury or disordered eating to cope when social supports (e.g., friends) and traditional mental health supports (e.g., school counselors) are no longer available, highlighting the need to think broadly and inclusively when considering strategies to support youth well-being (e.g., creating structures designed to enhance social connectedness in addition to increasing access to low-cost, high-quality mental health treatment).

Minority youth faced different levels of loss of basic needs and endorsed different rates of maladaptive coping during the pandemic. For instance, youth who identified as multi-racial/muti-ethic reported greater losses of basic needs-perhaps due to existing structural and institutional barriers (Assari, 2018; Burton et al., 2010). Racial and ethnic minority families historically live in socioeconomically disadvantaged areas (Burton et al., 2010). The pandemic required social distancing, inadvertently limiting access to certain services, resources, and products; it is likely that these limits were most severe in the least wealthy areas (often in densely-populated cities), exacerbating disadvantages and hardships among these areas' residents. The pandemic may have likewise exacerbated challenges for gender and sexual minority youth-particularly those forced to quarantine in home environments that dis-affirm or invalidate their identities (Roulston et al., under review). Pandemic-related stress may have resulted in greater reliance on maladaptive coping for these youth. Therefore, the stress of the pandemic increased such coping strategies (e.g., self injury, substance use, and disordered eating). Notably, participants identifying as Asian in this study reported engaging in maladaptive coping less than their White/Caucasian peers. Cultural norms in Asian communities (e.g., stronger familial relationships) may help explain this difference (Campos \& Kim, 2017).

Many adolescents reported using maladaptive coping strategies to cope with impacts of the pandemic, including eating less than usual ( $51 \%$ of adolescents), eating more than usual (49\% of adolescents), and non-suicidal self-injury ( $24 \%$ of adolescents). Indeed, a majority of youth in this sample endorsed one or more maladaptive coping strategies, potentially reflecting the universally stressful nature of the pandemic. The analyses demonstrated that youth who endorsed greater losses of basic needs were more likely to report maladaptive coping than were peers without such losses, but this association was relatively weak. This pattern suggests that loss of basic needs may be only one of many underlying factors related to maladaptive coping in youth. For example, pre-pandemic levels of psychopathology (e.g. anxiety and depression) or risk factors (e.g., hopelessness or self-dislike) may be exacerbated by events and challenges brought on by the pandemic (Dewa et al., 2021), in turn increasing reliance on maladaptive coping strategies like self-harm or drug use. Separately, pre-existing cultural differences-such as stigma surrounding discussion of mental illness-may preclude some youth from communicating their treatment needs to family members. Accordingly, youth may deem it necessary to find alternative (and perhaps unhelpful) ways to cope with their distress. Additionally, family risk factors predating the pandemic, such as high family conflict, place youth at greater risk for developing emotional and behavioral problems (Gervais \& Jose, 2020)—thus increasing risk for reliance on maladaptive coping strategies.

Some study limitations and further considerations should be noted. This study asked youth to report on coping behaviors at one time-point in the pandemic. Considering how coping behaviors might have changed over the course of the pandemic may elucidate longer-term impacts of losing access to food, housing, or school access as a result of the pandemic. Different state-level mandates (e.g., varying requirements for social distancing) and ongoing social and political challenges may have also influenced youth during this time. In addition, experiences reported by this sample may not generalize to youth in other countries. This study was only available in English which prevented non-English 
speakers from participating. While not a limitation, it is important to note that the results may not generalize to youth with low depression symptoms. However, it is of clinical importance to understand these trends in high risk youth.

Adolescents across the United States have experienced losses of basic needs-including housing and food insecurity-throughout the COVID-19 pandemic. Results of this study suggest that youth experiencing these losses reported greater engagement in maladaptive coping behaviors. Minoritzed youth reported losing more basic needs, and greater reliance on maladaptive coping behaviors, relative to their non-minoritized peers. It is imperative for those in contact with youth, including healthcare professionals and teachers, to actively assess loss of basic needs-and their potential behavioral consequences-during and beyond the COVID-19 pandemic. Environmental and structural factors (e.g., homelessness, food insecurity) might shape how educators and healthcare professionals structure support for the young people they serve. Notably, recommendations to 'change behaviors' or simply rely on more adaptive coping strategies, may be less helpful for youths with few alternative options due to challenges in their everyday environments. Lastly, there is a clear need to prioritize both restoration of basic needs and access to mental health supports for youth, particularly since the pandemic's impacts on well-being and safety may extend far after disease risk declines.

Funding Statement Research reported in this manuscript work was supported by the NIH Office of the Director under award number DP5OD028123 (PI: Schleider).

Author Contributions AS conceived of the study, participated in its design, performed statistical analysis and interpretation of data, and drafted the manuscript; JF participated in the design of the study, interpretation of data, and drafted the manuscript; KRF participated in the design of the study, interpretation of data, and drafted the manuscript; JLS acquired funding, participated in the design of the study and interpretation of data, and drafted the manuscript. All authors read and approved the final manuscript.

Data Availability Anonymized data that support the findings of this study are openly available on the Open Science Framework at https:// osf.io/3qtcb/.

\section{Declarations}

Conflict of Interest JLS receives grant support from the National Institutes of Health (DP5OD28123), the Klingenstein Third Generation Foundation, the American Psychological Foundation, the Society for Clinical Child and Adolescent Psychology, and Limbix, Inc. JLS and KRF receive grant support from the Upswing Fund for Adolescent Mental Health. JLS receives royalties from sales of a therapeutic workbook for adolescents, published by New Harbinger. JLS is under contract with Oxford University Press to co-edit a book on low-intensity mental health interventions for youth. The senior author receives grant support from the National Institutes of Health, the Klingenstein Third Generation Foundation, the American Psychological Foundation, the Society for Clinical Child and Adolescent Psychology, and Limbix,
Inc. The third and senior authors receive grant support from the Upswing Fund for Adolescent Mental Health. The senior author receives royalties from sales of a therapeutic workbook for adolescents, published by New Harbinger, and is under contract with Oxford University Press to co-edit a book on low-intensity mental health interventions for youth. The authors report no other financial conflicts.

Ethics Statement/Informed Consent Study procedures were approved by the University of Denver IRB prior to participant enrollment, and all adolescents provided online assent prior to beginning the study. In line with ethical guidelines, given that it is difficult if not impossible to ensure parent/guardian consent online (Smith et al., 2021), parent permission was not required to participate in this study; for this study, it was formally waived by the University of Denver IRB. This provided anonymity and minimized access barriers (e.g., discomfort disclosing psychological distress, as parents are often unaware of their adolescents' depressive symptoms, including suicidal and morbid ideation, in up to $80 \%$ of cases; Brahmbhatt \& Grupp-Phelan, 2019).

\section{References}

Assari, S. (2018). Unequal gain of equal resources across racial groups. International journal of health policy and management, 7(1), 1-9. https://doi.org/10.15171/ijhpm.2017.90

Bartholdy, S., Allen, K., Hodsoll, J., O’Daly, O. G., Campbell, I. C., Banaschewski, T., Bokde, A. L., Bromberg, U., Büchel, C., Quinlan, E. B., Conrod, P. J., Desrivières, S., Flor, H., Frouin, V., Gallinat, J., Garavan, H., Heinz, A., Ittermann, B., Martinot, J.-L., et al. (2017). Identifying disordered eating behaviours in adolescents: How do parent and adolescent reports differ by sex and age? European Child \& Adolescent Psychiatry, 26(6), 691-701. https://doi. org/10.1007/s00787-016-0935-1

Beccia, A., Baek, J., Jesdale, W., Austin, S., Forrester, S., Curtin, C., \& Lapane, K. (2019). Risk of disordered eating at the intersection of gender and racial/ethnic identity among U.S. high school students. Eating Behaviors: an International Journal, 34, 101299-101299. https://doi.org/10.1016/j.eatbeh.2019.05.002

Bell, K. A., Gratch, I., Ebo, T., \& Cha, C. B. (2021). Examining discrepant reports of adolescents' self-injurious thoughts and behaviors: A focus on racial and ethnic minority families. Archives of suicide research, 1-15. Advance online publication. https://doi. org/10.1080/13811118.2021.1925607.

Rich, B. (2021). table1: Tables of descriptive statistics in HTML. R package version 1.4. https://CRAN.R-project.org/package $=$ table 1

Bettis, A., Thompson, E., Burke, T., Nesi, J., Kudinova, A., Hunt, J., Liu, R., \& Wolff, J. (2020). Prevalence and clinical indices of risk for sexual and gender minority youth in an adolescent inpatient sample. Journal of Psychiatric Research, 130, 327-332. https:// doi.org/10.1016/j.jpsychires.2020.08.022

Blashill, A. J., Fox, K., Feinstein, B. A., Albright, C. A., \& Calzo, J. P. (2021). Nonsuicidal self-injury, suicide ideation, and suicide attempts among sexual minority children. Journal of Consulting and Clinical Psychology, 89(2), 73-80. https://doi.org/10.1037/ ccp0000624

Brahmbhatt, K., \& Grupp-Phelan, J. (2019). Parent-adolescent agreement about adolescent's suicidal thoughts: A divergence. Pediatrics, 143(2), e20183071. https://doi.org/10.1542/peds.2018-3071

Burton, L. M., Bonilla-Silva, E., Ray, V., Buckelew, R., \& Hordge Freeman, E. (2010). Critical race theories, colorism, and the decade's research on families of color. Journal of Marriage and Family, 72(3), 440-459. https://doi.org/10.1111/j.1741-3737. 2010.00712.x 
Campos, B., \& Kim, H. S. (2017). Incorporating the cultural diversity of family and close relationships into the study of health. The American Psychologist, 72(6), 543-554. https://doi.org/10. 1037/amp0000122

CDC. (2020). CDC COVID-19 community survey question Bank (DRAFT). U.S. National Library of Medicine. https://cde.nlm. nih.gov/formView?tinyId=Kcceysolt.

Chen, F., Zheng, D., Liu, J., Gong, Y., Guan, Z., \& Lou, D. (2020). Depression and anxiety among adolescents during COVID-19: A cross-sectional study. Brain, Behavior, and Immunity, 88, 36-38. https://doi.org/10.1016/j.bbi.2020.05.061

Corliss, H., Goodenow, C., Nichols, L., \& Bryn Austin, S. (2011). High burden of homelessness among sexual-minority adolescents: Findings from a representative Massachusetts high school sample. American Journal of Public Health, 101(9), 1683-1689. https://doi.org/10.2105/AJPH.2011.300155

Cutuli, J. (2018). Homelessness in high school: Population-representative rates of self-reported homelessness, resilience, and risk in Philadelphia. Social Work Research, 42(3), 159-168. https://doi.org/10.1093/swr/svy013

Dewa, L. H., Crandell, C., Choong, E., Jaques, J., Bottle, A., Kilkenny, C., Lawrence-Jones, A., Di Simplicio, M., Nicholls, D., \& \& Aylin, P. (2021). Ccopey: A mixed-methods coproduced study on the mental health status and coping strategies of young people during COVID-19 UK lockdown. Journal of Adolescent Health, 68(4), 666-675. https://doi.org/10.1016/j.jadohealth. 2021.01.009

Ellis, W. E., Dumas, T. M., \& Forbes, L. M. (2020). Physically isolated but socially connected: Psychological adjustment and stress among adolescents during the initial COVID-19 crisis. Canadian Journal of Behavioural Science, 52(3), 177-187. https://doi.org/10.1037/cbs0000215

Gelinas, L., Pierce, R., Winkler, S., Cohen, I. G., Lynch, H. F., \& Bierer, B. E. (2017). Using social media as a research recruitment tool: ethical issues and recommendations. The American Journal of Bioethics, 17(3), 3-14.

Gervais, C., \& Jose, P. E. (2020). How does family connectedness contribute to youths' health? The mediating role of coping strategies. Family Process, 59(4), 1627-1647. https://doi.org/ 10.1111/famp.12514

Gonzalez-Blanks, A., Bridgewater, J. M., \& Yates, T. M. (2020). Statistical approaches for highly skewed data: Evaluating relations between maltreatment and young adults' non-suicidal selfinjury. Journal of Clinical Child and Adolescent Psychology, 49(2), 147-161. https://doi.org/10.1080/15374416.2020.17245 43

Green, A., Bekman, N., Miller, E., Perrott, J., Brown, S., \& Aarons, G. (2011). Parental awareness of substance use among youths in public service sectors. Journal of studies on alcohol and drugs, 72(1), 44-52. https://doi.org/10.15288/jsad.2011.72.44

Klaus, N. M., Mobilio, A., \& King, C. A. (2009). Parent-adolescent agreement concerning adolescents' suicidal thoughts and behaviors. Journal of Clinical Child and Adolescent Psychology, 38(2), 245-255. https://doi.org/10.1080/15374410802698412

Kovacs, M. (2011). Children's depression inventory - 2 (2nd ed.). Multi-Health Systems Inc.

Kuhfeld, M., Soland, J., Tarasawa, B., Johnson, A., Ruzek, E., \& Liu, J. (2020). Projecting the potential impact of COVID-19 school closures on academic achievement. Educational Researcher, 49(8), 549-565. https://doi.org/10.3102/0013189X20965918

Henry, L. \& Wickham, H. (2020). Purrr: Functional programming tools. R package version 0.3.4. https://CRAN.R-project.org/ package $=$ purrr

Magson, N. R., Freeman, J. Y., Rapee, R. M., Richardson, C. E., Oar, E. L., \& Fardouly, J. (2021). Risk and protective factors for prospective changes in adolescent mental health during the
COVID-19 pandemic. Journal of Youth and Adolescence, 50(1), 44-57. https://doi.org/10.1007/s10964-020-01332-9.

McDermott, B., \& Cobham, V. (2014). A stepped-care model of postdisaster child and adolescent mental health service provision. European Journal of Psychotraumatology, 5(1), 24294-24210. https://doi.org/10.3402/ejpt.v5.24294

Mclaughlin, K. A., Fairbank, J. A., Gruber, M. J., Jones, R. T., Lakoma, M. D., Pfefferbaum, B., Sampson, N. A., \& Kessler, R. C. (2009). Serious emotional disturbance among youths exposed to hurricane Katrina 2 years post disaster. Journal of the American Academy of Child and Adolescent Psychiatry, 48(11), 1069-1078. https://doi.org/10.1097/CHI.0b013e3181 b76697

Oberle, M., Romero Willson, S., Gross, A., Kelly, A., \& Fox, C. (2019). Relationships among child eating behaviors and household food insecurity in youth with obesity. Childhood Obesity, 15(5), 298-305. https://doi.org/10.1089/chi.2018.0333

Phillips, G., Turner, B., Salamanca, P., Birkett, M., Hatzenbuehler, M., Newcomb, M., Marro, R., \& Mustanski, B. (2017). Victimization as a mediator of alcohol use disparities between sexual minority subgroups and sexual majority youth using the 2015 National Youth Risk Behavior Survey. Drug and Alcohol Dependence, 178, 355-362. https://doi.org/10.1016/j.druga lcdep.2017.05.040

R Core Team. (2021) R: A language and environment for statistical computing. R Foundation for Statistical Computing. https:// www.Rproject.org

Revelle, W. (2020) psych: Procedures for Personality and Psychological Research, Northwestern University, Evanston, Illinois, USA, https://CRAN.R-project.org/package $=$ psych Version $=$ 2.1.3.

Richardson, L. P., Rockhill, C., Russo, J. E., Grossman, D. C., Richards, J., McCarty, C., McCauley, E., \& Katon, W. (2010). Evaluation of the PHQ-2 as a brief screen for detecting major depression among adolescents. Pediatrics, 125(5), e1097-e1103. https://doi.org/10.1542/peds.2009-2712

Roulston, C., McKetta, S., Price, M., Fox, K., \& Schleider, J. L. (under review). Structural correlates of mental health support access among sexual minority youth of color during COVID-19. PsyArXiv, 1, https://doi.org/10.31234/osf.io/n8tm7.

Schaumberg, K., Reilly, E., Anderson, L., Gorrell, S., Wang, S., \& Sala, M. (2018). Improving prediction of eating-related behavioral outcomes with zero-sensitive regression models. Appetite, 129, 252-261. https://doi.org/10.1016/j.appet.2018.06.030

Schleider, J. L., Mullarkey, M.C., Fox, K.R., Dobias, M.L., Shroff, A., Hart, E.A., Roulston, C. (2021, in press). A randomized trial of online single-session interventions for adolescent depression during COVID-19. Nature Human Behaviour. Advance online publication.

Smith, D., Lipson, S., Wang, S., \& Fox, K. (2021). Online methods in adolescent self-injury research: Challenges and recommendations. Journal of Clinical Child and Adolescent Psychology, 1-12. https://doi.org/10.1080/15374416.2021.1875325.

Stogner, J., \& Gibson, C. L. (2011). The influence of health strain on the initiation and frequency of substance use in a national sample of adolescents. Journal of Drug Issues, 41(1), 69-93. https://doi.org/10.1177/002204261104100104

Tompsett, C., Domoff, S., \& Toro, P. (2013). Peer substance use and homelessness predicting substance abuse from adolescence through early adulthood. American Journal of Community Psychology, 51(3), 520-529. https://doi.org/10.1007/ s10464-013-9569-3

Venables, W. N., \& Ripley, B. D. (2002). Modern applied statistics with $S$ (4th ed.). Springer ISBN.

Watson, R. J., Adjei, J., Saewyc, E., Homma, Y., \& Goodenow, C. (2017). Trends and disparities in disordered eating among 
heterosexual and sexual minority adolescents. International Journal of Eating Disorders, 50, 22-31. https://doi.org/10. 1002/eat.22576

Wei, T., \& Simko, V. (2021). R package 'corrplot': visualization of a correlation matrix. (Version 0.90), https://github.com/taiyun/ corrplot.

Wei, T., Simko, V., Levy, M., Xie, Y., Jin, Y., \& Zemla, J. (2017). Corrplot: Visualization of a correlation matrix (0.84) [computer software]. https://CRAN.R-project.org/package=corrplot.

Wickham, H., Averick, M., Bryan, J., Chang, W., McGowan, L., François, R., Grolemund, G., Hayes, A., Henry, L., Hester, J., Kuhn, M., Pedersen, T., Miller, E., Bache, S., Müller, K., Ooms, J., Robinson, D., Seidel, D., Spinu, V., et al. (2019). Welcome to the
Tidyverse. Journal of Open Source Software, 4(43), 1686. https:// doi.org/10.21105/joss.01686

Wickham, H., Chang, W., Henry, L., Pedersen, T. L., Takahashi, K., Wilke, C., Woo, K., Yutani, H., Dunnington, D., \& R Studio. (2020). ggplot2: Create Elegant Data Visualisations Using the Grammar of Graphics (3.3.2) [Computer software]. https:// CRAN.R-project.org/package=ggplot 2 .

Publisher's Note Springer Nature remains neutral with regard to jurisdictional claims in published maps and institutional affiliations. 